\title{
Lung folding simulating peripheral pulmonary neoplasm (Blesovsky's syndrome)
}

\author{
C R PAYNE, P JAQUES, AND I H KERR
}

From the Brompton Hospital, London

ABSTRACT Six cases are reported in whom the diagnosis of benign pleural thickening with lung folding simulating peripheral pulmonary neoplasm was made. Three patients presented with chest pain, two were asymptomatic, and the abnormality was a chance finding in one patient with asthma. The radiographs in all cases showed similar appearances-a peripheral opacity appearing to lie within the lung, usually the lower lobe, and characteristic curvilinear shadows extending from the opacity to the hilum. At thoracotomy, predominantly visceral pleural thickening had caused the underlying lung to fold. Hyaline plaques were present on the parietal pleura elsewhere and pleural adhesions were usually absent. Adequate removal of the thickened visceral pleura in five patients allowed the folded lung to re-expand, with reversion of the chest radiograph to normal. One patient in whom the underlying lung folding was not appreciated at operation still has chest pain and the chest radiograph is unchanged. The radiographic appearances of this non-malignant condition, especially the appearances on lateral tomography, are diagnostic, and recognition may obviate the need for operation in asymptomatic patients. For those patients with chest pain, thoracotomy with removal of the visceral pleura and release of the folded lung appears to be effective in relieving this symptom.

Benign pleural thickening may occur in the form of localised hyaline plaques or generalised pleural disease covering one or more lobes. The affected pleura consists of glistening, cartilage-like areas of thickening on either the visceral or parietal layers. As organisation proceeds, infolding of the underlying lung occurs which on the radiograph produces the appearance of a peripheral mass and may mimic a peripheral carcinoma of the lung. ${ }^{1}$ Tomography, however, frequently reveals the presence of curvilinear shadows connecting the "mass" to the hilum of the lung. These shadows are not seen with peripheral pulmonary neoplasms. Volume loss may be apparent in the affected lobe. These signs are of value in the differential diagnosis of such cases.

\section{Patients}

Six cases are reported in whom the diagnosis of pleural thickening with lung folding was made at thoracotomy. Five patients were male and one (case 6) female. The age range was from 31 to

Address for reprint requests: Dr IH Kerr, Department of Radiology, Brompton Hospital, Fulham Road, London SW3 6HP.
60 years. Three patients presented with persistent, non-pleuritic chest pain and one with coincidental dyspnoea on exertion related to reversible airways obstruction. The remaining two cases were asymptomatic, having been discovered during mass miniature radiography surveys. There was no relevant occupational history and in particular, there was no known contact with asbestos. Four patients had physical signs compatible with pleural thickening. Sputum was examined in five patients and no acid-fast bacilli or malignant cells were found. Erythrocyte sedimentation rates ranged from 5 to $48 \mathrm{~mm}$ in one hour. Four of these cases underwent bronchoscopy. The appearances were entirely normal.

\section{Radiographic and surgical findings}

Each patient had frontal and lateral chest radiography, followed by relevant antero-posterior and lateral tomography. In all six cases a quite definite peripheral mass was identifiable, apparently situated within the lung but attached to the pleural surface. These patients underwent exploratory thoracotomy because of the radiographic features. 
CASE 1 (AM)

Plain radiographs showed a mass in the right lower lobe posteriorly and calcified tuberculous lesions in the left upper lobe (fig 1a). There was marked pleural thickening in the costophrenic recess. Lateral tomography demonstrated curvi-

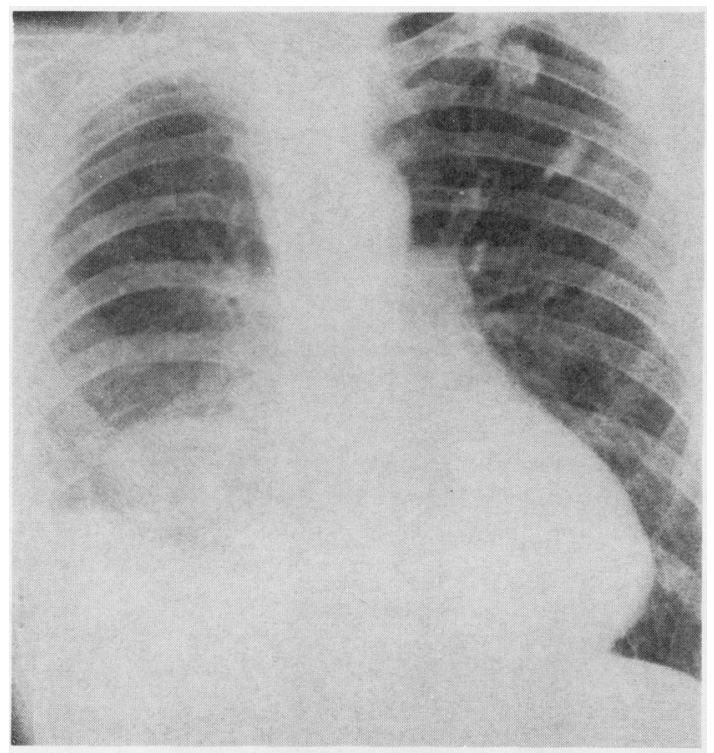

(a)

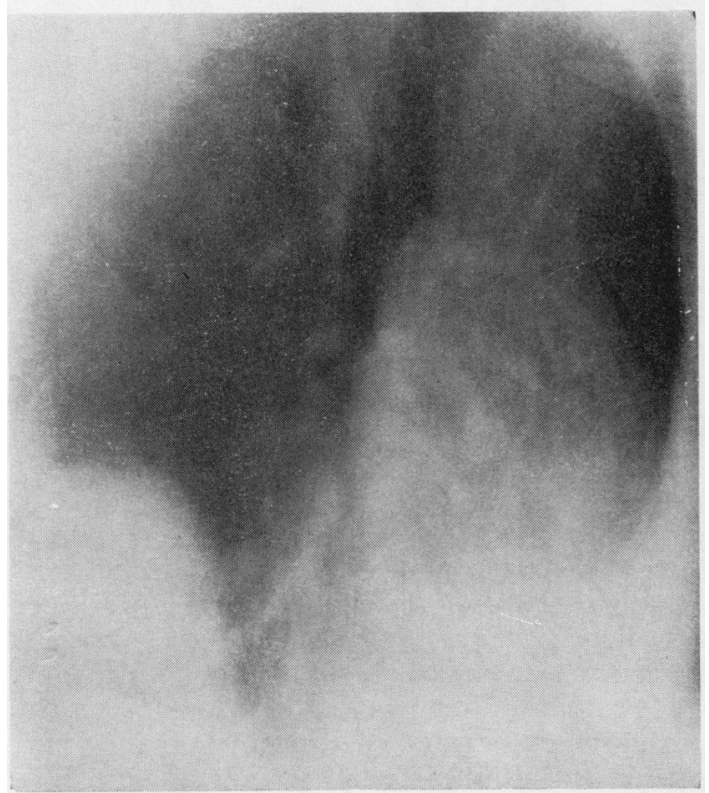

(b)

Fig 1 Case 1: (a) postero-anterior radiograph, (b) lateral tomograph showing right lower zone opacity, pleural thickening, and curvilinear shadows. linear shadows stretching from the mass to the right hilum (fig 1b). At thoracotomy the right lung was found to be encased in a thick fibrous coat of visceral pleura, most dense over the lower and middle lobes. The diaphragmatic surface of the right lower lobe had rotated upwards and posteriorly almost to the apex of the right lower lobe. There was $60 \mathrm{ml}$ of serous pleural fluid and minimal adhesions between visceral and parietal layers. After pulmonary decortication the right lower lobe expanded to its normal volume and shape. Histology of the thickened visceral pleura was reported as showing organised fibrin and prominent lymphoid follicles with no evidence of "collagen change."

\section{CASE 2 (DL)}

Radiography demonstrated an irregular opacity in the right lower lobe posteriorly, attached to the pleura and with curvilinear streaks radiating towards the hilum (fig 2). There was an abnormal density in the middle lobe region. Thoracotomy revealed grossly thickened visceral and parietal pleura encasing the middle and right lower lobes and $600 \mathrm{ml}$ of serous pleural fluid. The posterior part of the right lower lobe was folded. After decortication there was good reexpansion of the middle and right lower lobes. Histology of the excised pleura was fibrous tissue only, without asbestos bodies.

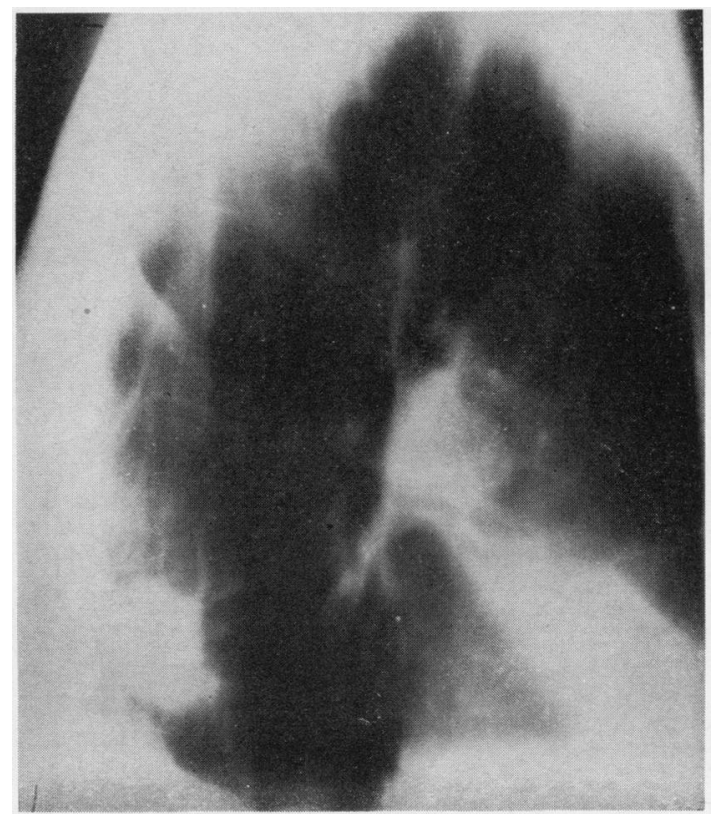

Fig 2 Case 2: lateral tomograph showing an opacity lying posteriorly in the right lower lobe. 
CASE 3 (GWB)

Radiographs of this patient showed a round opacity apparently lying in the periphery of the left lower lobe with curvilinear shadows connecting the opacity to the hilum (fig 3). At thoracotomy, thick smooth fibrous plaques were present in the paravertebral gutter, on the diaphragm, and on the lower lobe posteriorly, with infolding of the left lower lobe. Wedge resection of the folded lung segment with overlying pleura was performed. Histology showed severe focal, pleural, and interlobular fibrosis with no evidence of inflammation or neoplasm.

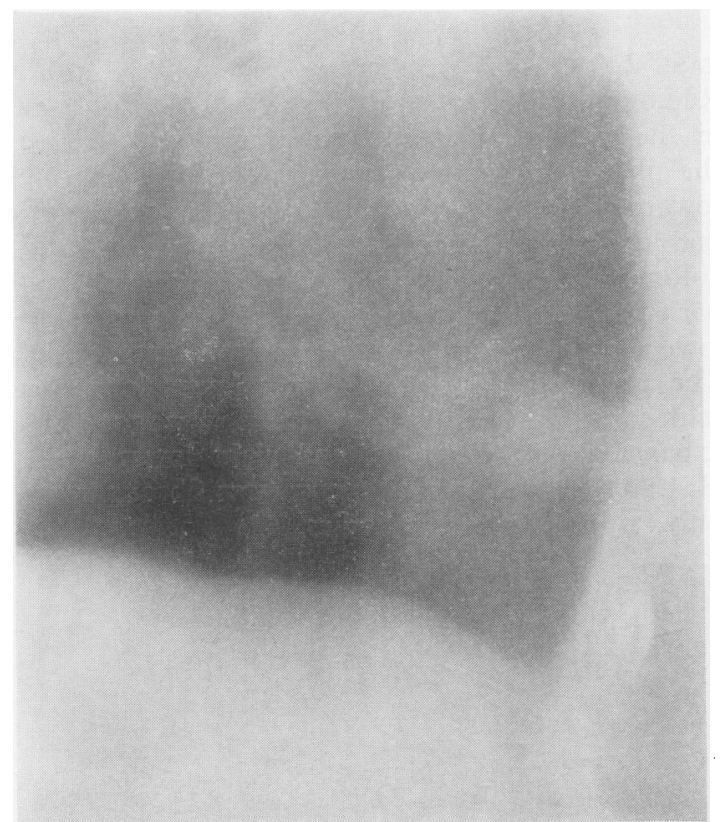

Fig 3 Case 3: lateral tomograph demonstrating opacity in left lower lobe with curvilinear shadows.

\section{CASE 4 (GD)}

Radiography demonstrated pleural thickening over the left lung with a $3 \mathrm{~cm}$ opacity in the left lower zone attached to the pleural surface. Curvilinear shadows extended from this region to the hilum (fig 4). At left thoracotomy the pleura was extremely thickened with extensive adhesions. The lingula had folded into the fissure, and smaller areas of folding were also present in the lower lobe. Pleurectomy was performed, releasing the areas of folded lung. Histology of the excised pleura showed marked fibrosis and foci of chronic inflammatory cells without evidence of malignancy.
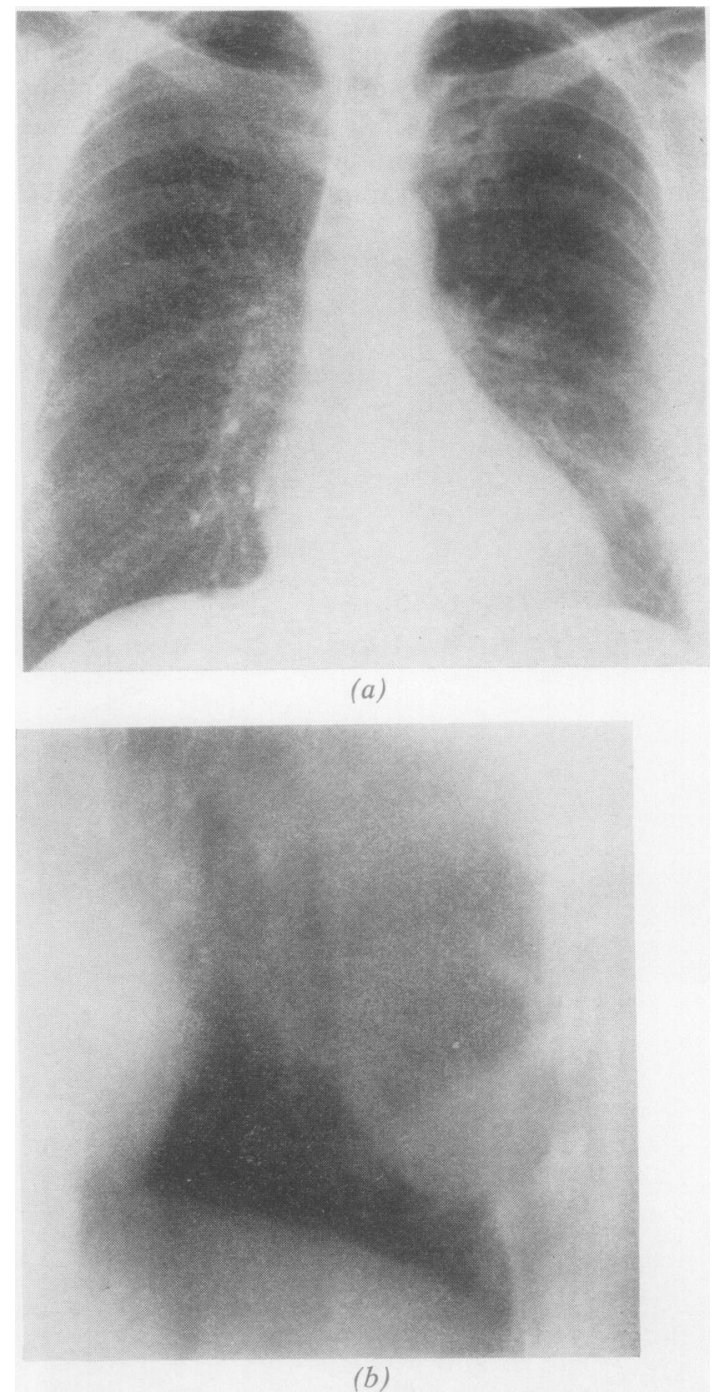

Fig 4 Case 4: (a) postero-anterior radiograph, (b) lateral tomograph showing pleural thickening, left lower lobe opacity and curvilinear shadows.

CASE 5 (GB)

Plain radiography showed a poorly defined $5 \mathrm{~cm}$ mass lying posteriorly in the right lower lobe with pleural plaques on both chest walls (fig 5a). Tomography confirmed the pleural thickening over the right lower lobe posteriorly with an oval shaped opacity extending forward into the lower lobe and curvilinear shadows radiating to the hilum (fig $5 \mathrm{~b}$ ). At thoracotomy, hard white plaques were present on the parietal pleura. The visceral pleura was abnormal over the greater and lesser fissures and tended to enfold the right 
lower lobe. This visceral layer was excised and the underlying lung allowed to expand. Histology showed fibrosis with groups of reactive mesothelial cells.

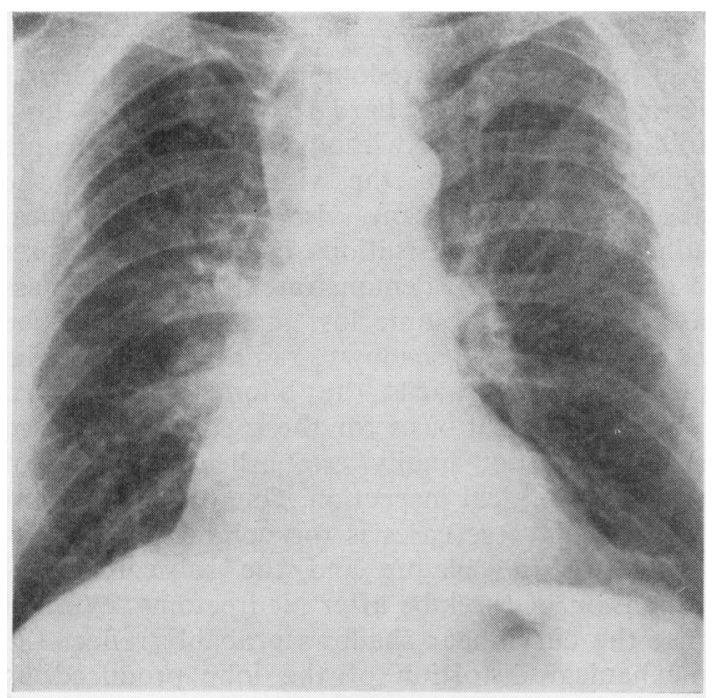

(a)

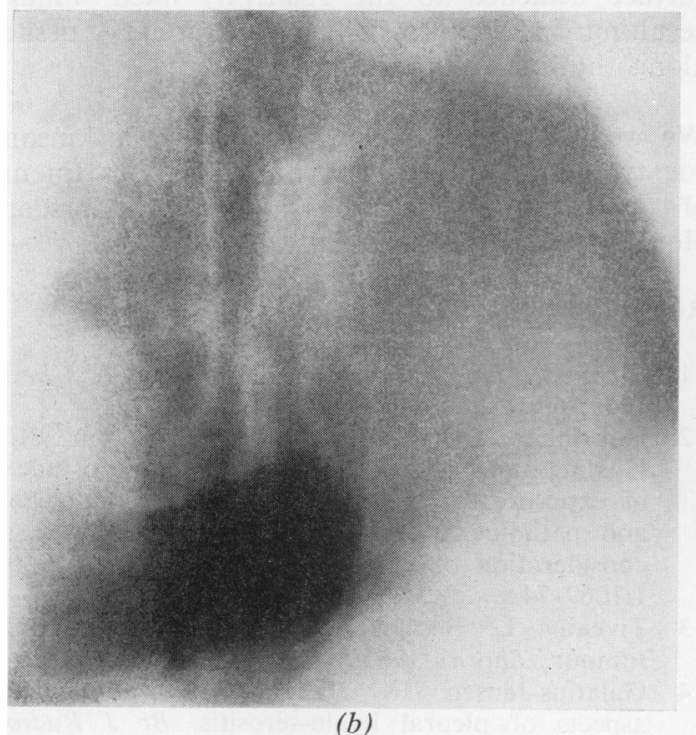

Fig 5 Case 5: (a) postero-anterior radiograph, (b) lateral tomograph showing right lower lobe opacity and curvilinear shadows radiating to the hilum. Marked volume loss of the right lower lobe is apparent.

CASE 6 (MW)

Frontal and lateral chest radiography showed an opacity lying posteriorly, apparently within the right lower lobe, with an associated pleural effusion (fig 6). Tomography confirmed the curvilinear shadows extending towards the hilum. Five hundred millilitres of sterile straw-coloured pleural fluid were aspirated before operation. Cytological examination of this fluid revealed lymphocytes and a few polymorphs. At right thoracotomy the visceral pleura was thickened over the posterior basal segment and adherent to the chest wall. Some areas of parietal pleural thickening were noted. Biopsies of parietal and visceral pleura showed dense fibrosis. Lung biopsy showed thickening of alveolar septa by fibrous tissue and foci of chronic inflammatory cells, predominantly lymphocytes. There was no evidence of malignancy. Pleurectomy and release of the underlying folded lung was not performed and postoperative chest radiographs still showed the opacity in the right lower zone with pleural thickening. This patient continued to complain of chest pain.

In summary all six cases showed discrete, peripheral, apparently intrapulmonary opacities with curvilinear shadows extending towards the hilum. The lower lobe was involved in all patients with additional folding of the lingular segment in one. All cases showed evidence of pleural thickening elsewhere and five had volume loss of the affected lobes.

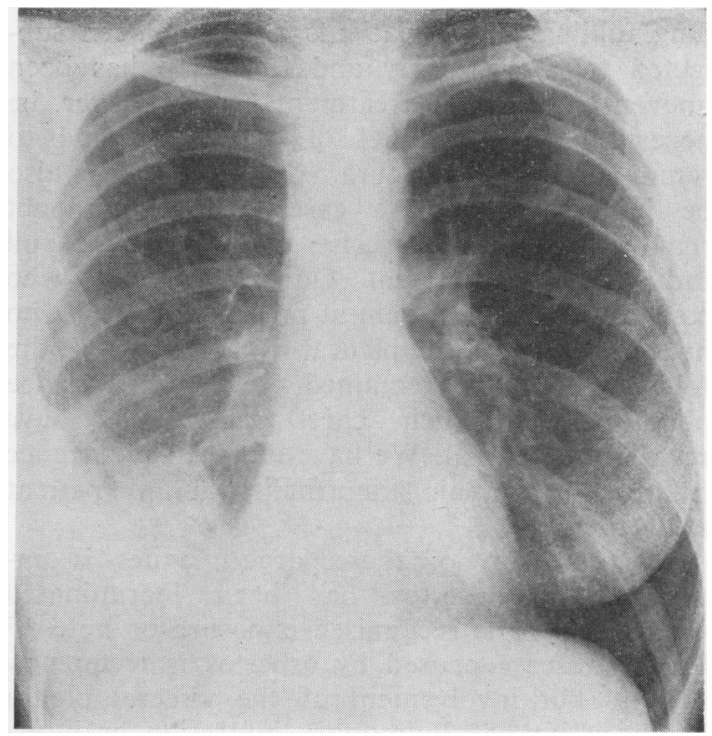

Fig 6 Case 6: postero-anterior radiograph demonstrating opacity in the right lower lobe, right pleural effusion, and curvilinear shadows. 


\section{Discussion}

Benign pleural plaques are an extremely common finding at necropsy, though only $15 \%$ of such cases have radiologically visible signs. ${ }^{2}$ Occasionally such plaques reach a size sufficient to make the radiologist uncertain as to whether he is dealing with a pleural or a penipheral pulmonary lesion. Tivenius drew attention to 10 such cases of benign pleural lesions simulating pulmonary tumours and recommended diagnostic pneumothorax as a means of localising the lesions to the pleura. ${ }^{3}$ In all his cases the pleural plaque was parietal although, as in our cases, there was a surprising lack of adhesions between the two pleural layers. This would have made diagnostic pneumothorax and thoracoscopy feasible. Two similar cases were described by Galatius-Jensen and Halkier who also advised diagnostic pneumothorax. ${ }^{4}$ Dobrowolski and Glowacka reported five cases, four of whom underwent thoracotomy before the diagnosis of benign hyaline pleural plaque was made. ${ }^{5}$ In these cases the plaque was associated with minimal adhesions and situated on the visceral pleura.

Our approach would stress the value of tomography. The appearances are so characteristic that a diagnosis can be made with some confidence and, therefore, operation can be avoided. Since we began to recognise this condition, we have seen several other asymptomatic cases with the characteristic radiographic appearances of lung folding. These patients have not been subjected to thoracotomy and follow-up has been uneventful. Surgical treatment may, however, be necessary for the relief of chest pain and to remove thickened pleura. Operation may also be indicated in those cases in whom doubt remains about the precise diagnosis after full radiographic assessment. Our two patients who complained of chest pain at presentation and who had removal of the pleura with release of the folded lung have remained free of pain since operation and their chest radiographs have reverted to normal. We have not seen recurrence of the radiographic abnormality in any patient after operation.

The aetiology of these large plaques is uncertain but asbestos has been incriminated whether there is recognised exposure or not. ${ }^{28} 7$ Pleural disease caused by asbestos is frequently parietal but involvement of the visceral pleura has been noted. ${ }^{2}$ Pulmonary infarction or infection is known on occasion to give rise to similar pleural thickening. The histopathology in either event is relatively nonspecific.
Blesovsky was the first to describe the "lung folding" which may occur in association with larger pleural plaques or grossly thickened pleural membrane. ${ }^{1}$ All three of his cases had a fibrous membrane on the visceral pleura without adhesions to the parietal layer. Our cases were also found to have predominantly visceral pleural lesions. Indeed, it is hard to visualise how lung folding could arise without the pleural plaque being primarily on the visceral layer in the absence of significant viscero-parietal pleural adhesions. The illustrations of Blesovsky's cases 2 and 3 elegantly demonstrate the pleural mass associated with volume loss in the adjacent lobe and curvilinear shadows radiating from the pleural mass towards the hilum of the lung. The pathological basis for these curiously curved shadows is not finally established, in spite of adequate surgical inspection. One of the constant features of these cases is the volume loss associated with the plaque and the subsequent reexpansion of the lobe after pleurectomy. We feel that the curvilinear shadows probably reflect the mechanical distortion of the lobe produced by the forcible contraction of the outer pulmonary surface attached to the relatively fixed hilum, resulting in compression of pulmonary tissue along the lines of the intralobar septa.

We would like to thank Miss Angela Parkinson for typing the manuscript and the Department of Medical Photography, Royal Marsden Hospital, for the illustrations.

\section{References}

1 Blesovsky A. The folded lung. Br J Dis Chest 1966; 60:19-22.

2 Hourihane DO'B, Lessof L, Richardson PC. Hyaline and calcified pleural plaques as an index of exposure to asbestos. A study of radiological and pathological features of 100 cases with a consideration of epidemiology. $\mathrm{Br}$ Med J 1966; 1:1069-74.

3 Tivenius L. Benign pleural lesions simulating tumour. Thorax 1963; 18:39-44.

4 Galatius-Jensen F, Halkier E. Radiological aspects of pleural hyalo-serositis. $\mathrm{Br} J$ Radiol 1965; 38:944-6.

5 Dobrowolski J, Glowacka R. Pleural hyaloserositis lesions imitating a lung tumour. Radiol Diagn (Berl) 1968; 9:199-205.

6 Collins TBF. Pleural reaction associated with asbestos exposure. Br J Radiol 1968; 41:655-61.

7 Kiviluoto R. Pleural plaques and asbestos: further observations on endemic and other nonoccupational asbestosis. Ann NY Acad Sci 1965; 132:235-9. 